\title{
LA CRISIS DE LAS \\ EMPRESAS INFORMATIVAS \\ REPLANTEA EL PERFIL \\ DEL PERIODISTA ACTUAL
}

Ana Gabriela Dávila J. ${ }^{1}$

\section{RESUMEN}

La revolución digital, a nivel global, y varios factores propios del contexto local, han transformado a las empresas informativas en todos los ámbitos que las componen. Uno de los cambios más importantes, involucra directamente al periodista, quien ha debido enfrentarse a nuevos escenarios en cuanto a la producción de contenidos, rutinas de trabajo y preferencias de las audiencias.

Este artículo expondrá y analizará los elementos que, a la luz del despunte tecnológico y del contexto nacional, han impulsado el pensar en un nuevo perfil profesional del periodista y, en ese sentido, se enfocará en delinear un perfil que abarque nuevas competencias y conocimientos.
Además, analizará la formación que históricamente han brindado las facultades de comunicación y/o periodismo del país y que definirá la convergencia y sus niveles como concepto fundamental para entender las transformaciones que han experimentado los medios de comunicación.

Palabras clave: medios de comunicación, convergencia, perfil del periodista, contenidos periodísticos.

\section{ABSTRACT}

The digital revolution, at a global level, and other factors belonging to the local context have transformed the mass media companies in all the fields they are a part of. One of the most important changes involves the journalists directly as they have had to face new scenarios

\footnotetext{
'Pontificia Universidad Católica del Ecuador, Facultad de Comunicación, Lingüística y Literatura, Quito, Ecuador (anagadavila@gmail.com).
} 
in which they deal with the production of contents, working routines and audience preferences.

This article will present and analyze the elements which, in light of technological advancement and the national context, have fostered the thought about finding a new professional profile. That is to say, it will focus on proposing a profile which encompasses new competencies and knowledge.
In addition, it will analyze the formation that has been offered by the schools of communication and/or journalism in the country for years and will define the convergence and its levels as a fundamental concept in order to understand the transformation that mass media has experienced.

Key words: mass media, convergence, journalist profile, journalistic contents

\section{INTRODUCCIÓN}

Los cambios y las transformaciones nunca han sido desconocidos para el periodismo. Su concepto, en sí mismo, se ha definido en función de la evolución de la historia del mundo. Basado en esto, se identifican tres etapas que tienen lugar en contextos específicos. La primera es la del periodismo ideológico, misma que llegó hasta el fin de la Primera Guerra Mundial; esta se caracterizó por impulsar un periodismo al servicio de ideas políticas y religiósas. Hacia 1870 nace la etapa del periodismo informativo, la que se destacó por el relato de los hechos más que por la difusión de comentarios. A través de esta, se daba cuenta de los principales acontecimientos que empezaron a transformar el mundo. A partir de 1945, cuando termina la Segunda Guerra Mundial, se gesta el periodismo interpretativo, caracterizado por una profundización de las informaciones, combinado con el punto de vista del periodista. Es durante este período cuando desde los medios se empezó a contribuir con elementos que permitían no solo conocer la realidad sino comprenderla.

Estas etapas trajeron consigo cambios tanto en el manejo de la información y del desarrollo del oficio periodístico, como en la forma del consumo de los productos periodísticos por parte de las audiencias. De ser un público que únicamente conocía el hecho o el qué, pasó a conocer las razones y los contextos, en otras palabras, el porqué de los acontecimientos.

Así se puede resumir, brevemente, las principales transformaciones que el periodismo ha experimentado a lo largo de su historia, hasta llegar a la revolución 
digital; para muchos la etapa que más cambios ha traído a los profesionales de la comunicación, principalmente, por el aparecimiento de las nuevas tecnologías de la información y comunicación.

En el mundo entero se habla de una crisis de la profesión, provocada por los cambios que están enfrentando las empresas informativas y que acarrea consigo la imperiosa necesidad de generar variados perfiles que puedan desenvolverse en una nueva realidad comunicativa e informativa, que plantea como cambio transcendental un nuevo modelo de comunicación.

\section{LA CRISIS DE LA PROFESIÓN}

Para entender la crisis de la profesión es preciso referirse a la crisis de las empresas informativas. Fruto de la revolución digital, los medios de comunicación han experimentado una serie de transformaciones que les han afectado tanto en su parte comercial como en la editorial. Los cambios en los contenidos, en las rutinas de producción, en los comportamientos de las audiencias, en la publicidad y en los modelos de negocios, han roto los esquemas tradicionales de manejo de los medios, lo cual también ha afectado a los periodistas. Para comprender lo apuntado cabe ir analizando cada uno de estos elementos relacionándolos con la actividad y función del periodista.

\section{Cambios en los contenidos}

Trabajar contenidos multiplataforma y multimediales requiere el conocimiento de las nuevas tecnologías. Ya no basta con escribir adecuadamente un texto y escoger una fotografía o ima- gen para acompañarlo. Se requiere manejar programas de edición de audio, video, fotografía, saber construir contenidos específicos para un teléfono o una tableta, por ejemplo. Se necesita, además, dotar a estos textos de elementos que generen interactividad y tengan hipertextos. También se deben trabajar contenidos de alta calidad informativa y contar buenas historias que atraigan a los lectores y sobresalgan dentro del océano de información que es la web. Es decir, se requieren conocimientos sólidos en varias herramientas digitales y nuevas narrativas, entre otros, los mismos que muchos periodistas no los tienen. Es ahí donde surge el primer inconveniente para el periodista, si no posee estos conocimientos podrá ser desplazado por quien tenga un perfil polivalente que es el que demandan los medios hoy.

\section{Rutinas de producción}

Los entornos digitales requieren 
un trabajo de 24/7; actualizar permanentemente la información y producir contenidos que serán distribuidos a través de distintos canales. Eso implica adaptar el mismo contenido al canal, con todo el esfuerzo que eso supone; algo parecido a trabajar tres y cuatro veces más. "El cambio en la estructura externa de los escenarios productivos tiene prolongación a los ciclos de organización del trabajo periodístico: los temarios se renuevan o actualizan en ciclos cada vez más cortos imbuidos de las dinámicas de actualización constante en Internet." (Tuñez, Martínez, Abejón 2010).

Antes, el reportaje sobre la delincuencia en la ciudad se lo trabajaba para publicarse en el periódico impreso, ahora ese contenido debe adaptarse, además, para aparecer en el periódico virtual, en la revista del grupo, en el video que se colgará en Facebook y en la galería de fotos que se publicará en Instagram. Cada producto debe ser elaborado tomando en cuenta las características propias del canal, es decir, es como crear un nuevo y diferente contenido cada vez. Si antes el trabajo del periodista era extenuante, ahora lo es más por la transformación de las rutinas de producción. En las redacciones actuales no se puede hablar de que la información es un producto terminado; hoy la información es un insumo para un producto que está en constante construcción (Franco, Pellicer 2014).

\section{Comportamiento de las audiencias}

Los periodistas ya no son los únicos que generan contenido para los medios, ahora son los públicos quienes también pueden contribuir con información, alterando así la dinámica de construcción de la información en la que solo había espacio para el periodista."Internet ha democratizado la posibilidad de convertirse en emisor para una audiencia transnacional que ha alimentado el papel de la audiencia como proveedor de productos propios y de productos elaborados a partir de lo transmitido por otros medios" (Tuñez et al 2010). En ese sentido, el papel de mediadores de la realidad puede haberse debilitado. ¿Cómo actuar frente al hecho que personas sin preparación específica, puedan también informar? De ahí surge el cuestionamiento sobre la formación que debe recibir un periodista. No es lo mismo publicar una foto y escribir un texto, con lo que de alguna manera se informa, que hacer un trabajo metodológico de búsqueda y procesamiento de información, de selección cuidadosa de fuentes, de análisis de contenidos, de reflexión de contextos, tareas indispensables del oficio periodístico y que deben seguir aprendiéndose en las escuelas de comunicación. A esto se suma, el enfrentarse a audiencias cada vez más heterogéneas, con amplio acceso a la información y saturadas de mensajes, que demandan información con valor agregado. 


\section{Publicidad y modelos de negocio}

Históricamente el sustento de los medios de comunicación ha sido la publicidad. La cantidad de usuarios que han sumado los medios digitales los han transformado en espacios atractivos para la publicidad, por lo que un gran porcentaje de esta se trasladó a ellos, dejando a los medios tradicionales sin piso sobre el cual sostenerse. Es pertinente también referirse, en este punto, a la crisis económica mundial que ha reducido los gastos en publicidad." EI descenso en las inversiones publicitarias es consecuencia directa de la situación mundial de crisis económica, pero en el ámbito de las empresas de comunicación coincide con la multiplicación de las plataformas de emisión que suponen los nuevos canales de televisión digital y con un constante desvío de recursos de los soportes tradicionales en papel a los soportes masivos on-line". (Tuñez et al 2010).

Internet trajo consigo la cultura de la gratuidad y con ella un cambio de paradigma en torno al consumo de productos informativos. ¿Por qué pagar por consumir un contenido si se puede hacerlo gratuitamente? De este cuestionamiento nace uno de los traspiés que han tenido que afrontar las empresas informativas: reformular su modelo de negocio. Tomemos el ejemplo de los medios impresos, su modelo de negocio tradi- cional planteaba tres vías por las que obtenían ingresos: la publicidad, las suscripciones y la venta de ejemplares. Si la publicidad se ha reducido y la venta de ejemplares también, ya que las audiencias prefieren consumir información a través de los soportes digitales en los que no pagan por informarse, ¿qué ingresos están recibiendo los medios impresos? El camino más inmediato para afrontar esta realidad ha sido poner en marcha nuevos modelos de negocio que aún no terminan de cuajar del todo. Uno de los más usados es cobrar por el acceso a cierto contenido con valor agregado. Otro modelo plantea acceder a un número limitado de información, y a partir de una cantidad específica de artículos leídos, cobrar para continuar consumiendo otros. Hay modelos que plantean, incluso, cobrar por otro tipo de servicios relacionados con el entorno digital, pero que no tienen que ver necesariamente con la producción de información periodística, sino más bien con la creación de contenido de otra especie.

Para los medios aplicar estos modelos de negocio ha significado una apuesta a ciegas, ya que ha sido muy difícil prever si van a ser efectivos o no. Al respecto, los empresarios de los medios reconocen que nadie tiene la receta del modelo perfecto; todo ha sido una experimentación a prueba y a error. 
La falta de ingresos, ineludiblemente, pone al medio en una situación de inestabilidad, misma que gatilla decisiones muchas veces extremas como la disminución de personal o la reducción en gastos de producción. En el Ecuador muchos periodistas se han visto afectados por esta situación y ahora se encuentran engordando las listas del desempleo. A diciembre del 2015 el porcentaje de desempleo en Ecuador fue de $4,77 \%$, de esa cifra, una parte corresponde a los periodistas despedidos de los medios de comunicación, dato que todavía no se ha contabilizado (www. periodismoecuador.com).

A estos elementos analizados, y que pueden ser extrapolados para entender la realidad de las empresas informativas en otros rincones del mundo, se suman otros propios del contexto local. Desde el 2007, año en que Rafael Correa asumió la presidencia del Ecuador, se han generado varios hechos que han marcado la relación prensa-poder, la misma que se ha desgastado al punto de afectar a la estabilidad de las empresas informativas. El juicio en contra de diario El Universo por el editorial No a las mentiras de Emilio Palacios; el juicio en contra de los periodistas Juan Carlos Calderón y Christian Zurita, autores del libro El gran hermano, investigación periodística en la que se develaba la supuesta participación de Fabricio Correa, hermano del Presidente de la República, en contratos millonarios con el gobierno; la creación de la Ley de Comunicación; la conformación de instancias reguladoras de la comunicación e información como la Secretaría de Comunicación (SECOM), la Superintendencia de la Información y Comunicación (SUPERCOM) y el Consejo de Regulación y Desarrollo de la Información y Comunicación (CORDICOM) y las permanentes acusaciones por parte del Presidente de la República en contra de los medios de comunicación cuestionando su manejo editorial y su función social. Todos estos estos hechos han generado un contexto complejo para ejercer la profesión en el país. Como hitos dentro de esta etapa se pueden mencionar el cierre de diario Hoy, el cambio de dueños de diario El Comercio, uno de los diarios referentes del país-actualmente está en manos de un empresario mexicano propietario de varios medios en Latinoamérica-y el despido de periodistas quienes han optado por crear sus propios medios. En la búsqueda de una salida para continuar ejerciendo la profesión y hacer frente al desempleo, varios periodistas han mirado en los medios digitales una alternativa. Según un estudio realizado por CIESPAL, en el 2012 existían 34 medios digitales en el país. Tres años más tarde, un mapeo desarrollado por Fundamedios, reveló que hasta abril del 2015, de los 34 medios solo quedaban 15. "Se hace periodismo en internet desde las cuatro regiones del país, con equipos mínimos o que pasan 
la docena, con recursos ajustados y deudas, pero con esfuerzos extraordinarios: más de uno ha pasado por la tentación de cerrar" (www.planv.com). De esta manera, los estudios muestran que algunos medios han logrado sobrevivir, pero otros no han tenido la misma suerte.

Este panorama local, además, ha llamado la atención de los organismos internacionales defensores de la libertad de expresión, quienes se han referido al país como un territorio complejo para ejercer el periodismo libremente. Con todos estos antecedentes, cabe la pregunta, ¿será el periodismo una buena profesión para ejercer en Ecuador?
"El momento de crisis que viven las empresas informativas, se suma a crisis de credibilidad de la profesión y al debilitamiento del papel de los periodistas en nuestra sociedad. El papel de mediadores de la realidad se está agotando tal como lo entendíamos décadas atrás. El retroceso de la profesión tal y como la vemos actualmente es triple: económico, de prestigio y de rol en la sociedad" (Franco, Pellicer 2014). Con todo lo expuesto se explica por qué la crisis de las empresas informativas es también la crisis del periodismo.

\section{LA CONVERGENCIA: CONCEPTO TEÓRICO PARA COMPRENDER LOS CAMBIOS EN LAS EMPRESAS INFORMATIVAS}

Desde la perspectiva teórica existe un concepto fundamental para comprender las transformaciones que han experimentado los medios de comunicación, a partir de la revolución digital, se trata de la convergencia. Esta no es consecuencia del surgimiento de Internet, pero es indiscutible que su aparición ha dado un impulso al concepto. Aunque ya existían diferentes formas de convergencia en los medios tradicionales, es innegable que el nuevo medio potenció y enriqueció el fenómeno. (Irigaray, Ceballos, Manna 2013). Existen varias definiciones del concepto de convergencia, una de ellas es la introducida por Henry Jenkins -investigador que más ha teorizado sobre este concepto- mencionada en el artículo Contenidos periodísticos en el ecosistema líquido, entre la convergencia y la divergencia, publicado en el libro Webperiodismo en un ecosistema líquido, quien la define como: "the flow of content across multiple media platforms, the cooperation between multiple media industries, and the migratory behavior of media audiences who will go almost anywhere in search the kind of entertainment they want". Con esta definición, el autor identifica tres 
grandes áreas en la convergencia: la distribución de información multiplataforma, la cooperación entre empresas y el comportamiento migratorio de las audiencias (Irigaray et al. 2013). Otro concepto abanderado por el investigador de nuevos medios, el español Ramón Salavarría, por su parte, apunta que la convergencia es un proceso multifactorial, e identifica cuatro componentes fundamentales que definen su marco estructural: convergencia tecnológica, profesional, empresarial y de contenidos (Irigaray et al. 2013).

\section{Convergencia empresarial}

Se deriva de la evolución del modelo de negocio de las empresas periodísticas. A partir de un determinado momento, la evolución tecnológica obligó a las empresas a un replanteamiento de sus estrategias, buscando rentabilizar sus inversiones en tecnología y recursos humanos en un escenario donde Internet emergía como medio del futuro. Las fusiones entre empresas y la estrategia de los grupos de comunicación de ampliar su oferta a todas las áreas - prensa, radio, televisión e Internet- generó grandes conglomerados mediáticos que buscaron en la cross-promotion formas de rentabilizar sus inversiones.

Este proceso es facilitado por la conexión que siempre existe entre nue- vos medios y sus antecesores, porque los nuevos medios renuevan los anteriores en un proceso de remediación. El nuevo medio es siempre una mejora, más o menos profunda, de los precedentes y, por ello, hay una dependencia entre los dos y existe un conjunto de características comunes que facilitan el proceso de convergencia en las empresas (Irigaray et al. 2013).

\section{Convergencia profesional}

Es consecuencia directa de la convergencia empresarial. Esta ya ocurría anteriormente, sobre todo, entre los periodistas que trabajan en radio y televisión, pero ganó más visibilidad con la digitalización. La simplificación técnica y la aceleración del proceso de producción periodística fueron aprovechados por las empresas para unir funciones: los fotógrafos, tal como los editores de sonido y de video, han visto desaparecer muchos puestos de trabajo porque las empresas imputaron esas ocupaciones a los periodistas. Cuánto más evolucionan los medios, mayor es la tendencia para que se establezca la polivalencia de los profesionales, que van acumulando funciones anteriores con las nuevas. (Irigaray et al. 2013).

\section{Convergencia tecnológica}

Los medios han sido empujados hacia un modelo de producción multi- 
plataforma porque los usuarios consumen cada vez más los contenidos en diferentes soportes o canales. Las nuevas demandas de los nativos digitales $y$, simultáneamente, de una audiencia móvil, han fragmentado el consumo, exigiendo a las empresas un reposicionamiento capaz de responder a los nuevos desafíos generados por esta audiencia liquida caracterizada por una conexión a la red permanente, universal y dinámica (Irigaray et al. 2013).

\section{Convergencia de contenidos}

Involucra a la producción de información multimedia que explota las características de las nuevas plataformas de recepción. En este caso se entiende la multimedialidad en su concepto más amplio, no solo la utilización de contenidos de diferentes tipologías -texto, sonido y video- en una unidad informativa homogénea y coherente, sino también en su mezcla con la hipertextualidad e interactividad (Irigaray et al. 2013).

El concepto de convergencia es uno de los más estudiados dentro del campo de las investigaciones sobre los medios. Relacionados con este concepto están el transmedia y el crossmedia, otros fenómenos propios de la cultura digital desde los que también se puede entender el rol actual del periodista como un creador de historias concebidas para ser expuestas a través de múltiples canales.

\section{LA FORMACIÓN DEL PERIODISTA}

Los cambios que se han suscitado en las empresas informativas, traen consigo, inevitablemente, un replanteamiento del perfil profesional del periodista. Antes de esbozar ideas que intenten construir ese perfil, conviene referirse a la formación que los periodistas han recibido, históricamente, en el país.

Primero cabe señalar que el proceso de profesionalización del periodista ecuatoriano se inició de manera formal dentro de las aulas universitarias en la década del 40, con la creación de la primera Facultad de Comunicación Social de la Universidad Central del Ecuador (1943) en Quito, y luego, con la formación de la Escuela de Información de la Universidad Estatal de Guayaquil, en Guayaquil (1945). Actualmente, se estima que en el país existen, aproximadamente, 26 escuelas y facultades de Comunicación Social.

María Isabel Punín, profesora investigadora de la Universidad Técnica Particular de Loja, señala en su trabajo titulado Los estudios de Comunicación Social/Periodismo en el Ecuador. Una visión crítica al rol de la universidad y la acade- 
mia que la enseñanza del periodismo en el país ha vivido dos momentos. El primero estuvo marcado por la ideología de izquierda, bajo la que los periodistas se formaban desde una óptica marxista. "Dentro de esa formación existió la idea de usar los medios desde la visión de la comunicación contestataria que combatió al sistema dominante" (Punín, 2012).

El segundo momento habría estado marcado por la tendencia neoliberal que dominó al mundo; es dentro de esta etapa cuando se estudian las relaciones públicas, la comunicación organizacional y el periodista recibe una formación apolítica (Punín, 2012). En el mismo estudio se señala que hasta la década del 60 existió una titulación pura en periodismo, la misma que luego cambió a partir de un proyecto realizado por el Centro Internacional de Estudios Superiores de Comunicación para América Latina, CIESPAL, el cual planteaba la formación polivalente del periodista, lo que permitió ampliar el perfil profesional y motivar la creación de más facultades y escuelas de comunicación en distintos países de la Región.

El periodismo desde sus inicios, ha sido una disciplina ecléctica que se ha nutrido de un sinnúmero de perspectivas teóricas y metodológicas. Esta posición multidisciplinaria, sumada a las tendencias del mercado, ha provocado que la formación de los comuni- cadores tenga dos tendencias limitadas. La primera, una formación de corte humanística, que considera al periodista un intelectual, y la segunda, la formación técnica sobre temáticas relacionadas con la producción audiovisual, el marketing y la comunicación organizacional (Punín, Martínez, 2013).

En América Latina, en tanto, el origen de la formación universitaria de comunicadores sociales puede situarse entre 1934 y 1935, cuando se empezaron a impartir cursos de periodismo a nivel superior en la Universidad de La Plata (Argentina); fue esta la primera universidad de la Región en ofertar la carrera. Las escuelas que nacieron durante este tiempo imitaron el modelo clásico que se estaba aplicando en las escuelas de periodismo de Estados Unidos y Europa. Según la Federación de Facultades Latinoamericanas de Comunicación, FELAFACS, existen 295 escuelas y facultades de comunicación en Latinoamérica (Punín et al. 2013).

Según el estudio de Punín, la formación de comunicadores en América Latina ha tenido tres momentos:

- Entre 1930 y 1960 corresponde el período en el cual los académicos se centran en el análisis de los medios, la producción informativa y el comportamiento de las audiencias. 
- Entre 1945 y 1969 se crea y se consolida CIESPAL, cuyo aporte fue proponer la formación polivalente de los comunicadores y plantear que las escuelas deberían tener un nivel académico, que la carrera debería tener una duración mínima de cuatro años e incluir en su programa de estudio enfoques humanísticos y técnico-profesionales.

- La década de los setenta es la etapa en la que los centros de for- mación universitaria trabajan en especializaciones y en la consolidación de los centros dedicados a la investigación (Punín et al. 2013).

En los últimos años la formación de los comunicadores ha tenido distintos enfoques, según la universidad. A nivel de las universidades que ofrecen la carrera en Quito, la clasificación es la siguiente:

\section{Tabla 1: Enfoques formativos de las carreras de Comunicación en las universidades que ofrecen la carrera en Quito}

\begin{tabular}{|c|c|}
\hline UNIVERSIDAD & ENFOQUE \\
\hline Universidad Central del Ecuador & Hacia el campo teórico de la comunicación. \\
\hline $\begin{array}{l}\text { Pontificia Universidad Católica } \\
\text { del Ecuador }\end{array}$ & $\begin{array}{l}\text { Hacia la literatura y la división clásica de los } \\
\text { medios: prensa, radio y televisión. También } \\
\text { cuenta con una mención en Comunicación } \\
\text { Organizacional. }\end{array}$ \\
\hline Universidad Politécnica Salesiana & Hacia la comunicación para el desarrollo social. \\
\hline Universidad de las Américas & $\begin{array}{l}\text { Hacia el campo organizacional de la } \\
\text { comunicación. }\end{array}$ \\
\hline $\begin{array}{l}\text { Universidad Internacional } \\
\text { del Ecuador }\end{array}$ & $\begin{array}{l}\text { Hacia el campo organizacional de la } \\
\text { comunicación. }\end{array}$ \\
\hline $\begin{array}{l}\text { Universidad Tecnológica } \\
\text { Equinoccial }\end{array}$ & Hacia las relaciones públicas y la publicidad. \\
\hline Universidad San Francisco de Quito & Hacia la comunicación multimedia. \\
\hline Universidad Internacional SEK & $\begin{array}{l}\text { Hacia los medios y la comunicación } \\
\text { organizacional. }\end{array}$ \\
\hline
\end{tabular}

Fuente: Ana Gabriela Dávila

Cabe anotar que en Quito la Universidad de los Hemisferios y la Universidad Técnica Particular de Loja también ofrecen una carrera en Comunicación, cuyo enfoque no difiere mayormente de los de las otras universidades citadas. 
De esta manera, al revisar lo que ofrecen las facultades de comunicación ubicadas en Quito, se puede resumir, a breves rasgos, la formación que han recibido los periodistas ecuatorianos en los últimos setenta años, tomando en cuenta que la mayoría de ellos se han formado en estas facultades, al ser las más representativas del país. Es preciso recordar que la facultad más antigua de comunicación del Ecuador tiene 73 años.

A partir de los cambios que han experimentado la comunicación y los medios, fruto de la revolución digital, en la mayoría de universidades a los planes de estudio que están basados en los en- foques descritos, se han incorporado materias relacionadas con las nuevas tecnologías. Sin embargo, como reconocen los docentes de varias universidades, en el escenario actual es inviable seguir sosteniendo el modelo de formación que planteaba el estudio desde la división clásica de los medios: prensa, radio, televisión. Hoy, es necesario pensar en otros ámbitos de acción para el periodista que están mucho más allá de los medios. Las facultades de comunicación, en la actualidad, tiene el reto de formar a los periodistas que deben conservar el ADN de la profesión, combinándolo con las nuevas herramientas y lenguajes que ofrece el campo digital.

\section{EL NUEVO PERFIL PROFESIONAL}

En las facultades de comunicación del país y del mundo se encuentra instaurado el debate en torno a cómo debería ser el perfil del periodista actual. El problema que se enfrenta desde la academia es que existe una brecha entre lo que se imparte y lo que el campo laboral demanda. La revolución digital ha traído consigo nuevos perfiles que fusionan el conocimiento técnico de las herramientas digitales, con el manejo de las prácticas del oficio periodístico tradicional, entre otros saberes; perfiles que hace cinco años no existían en los medios y que no están contemplados en ningún plan de estudio de las facultades que ofertan la carrera.
Uno de los grandes problemas de las facultades de Comunicación actuales es que se enfrentan a una brecha casi insalvable con la realidad. Se enseña mucho para los problemas del pasado pero se aprende poco de los desafíos del futuro. Y no se debe a una especial incompetencia educativa, simplemente es complicado que propuestas docentes y planes de estudio vayan al ritmo de las tecnologías de la comunicación. En otras palabras y bajo la perspectiva más pragmática (no útil) de la universidad, esta tiene el difícil reto de preparar a los futuros profesionales de la comunicación para muchas profesiones que aún no existen". (Noguera, Martínez, Grandío, 2011) 
Prueba de esto son los anuncios que se publican requiriendo personal en los que aparecen cargos como editor web, periodista de datos, estratega de medios digitales, especialista en narrativas digitales, consultor digital, periodista multimedia, entre otros. Aunque no se detalle, específicamente, los conocimientos que deben tener, se sabe que debe ser alguien que conozca bien las herramientas digitales y la cultura digital. Es decir, no basta con manejar bien Facebook, Twitter o Instagram, sino que entienda las dinámicas que rigen el espacio digital, los intereses de las audiencias, la lógica y códigos de la comunicación digital. Al no encontrar profesionales que integren en su perfil estos conocimientos, ya que las universidades no los ofrecen, se buscan personas que, por ejemplo, sean curiosas, les guste experimentar en nuevos ámbitos, estén interesados en explorar a través de Internet, disfruten compartiendo contenidos en redes sociales, generen con frecuencia contenidos para sus "blogs", es decir, que tengan una actitud positiva frente a la tecnología más que conocimientos específicos. En ese sentido, en esos perfiles se estaría haciendo más hincapié en las actitudes que en las aptitudes. Es preciso señalar, además, que estos perfiles podrían estar en cualquier facultad, sin embargo, el mercado los busca en las facultades de comunicación ya que el componente básico de los procesos que se desarrollan desde estos cargos, es la comunicación (Franco, Pellicer 2014).
Ser una persona versátil, que se adapte fácilmente a distintos entornos y que esté abierta a los cambios, es una de las actitudes que puede ser más útil para desempeñarse en los medios de hoy en donde los cambios transcurren vertiginosamente. En el libro Optimismo para periodistas: claves para entender los nuevos medios en la era digital, se recoge lo que señala que Steve Hermann, editor de la web de la BBC NEWS, quien manifiesta que los periodistas deben "amar" el cambio. "Si no te gusta el cambio vas a ser una persona muy estresada e infeliz, porque las cosas cambian muy rápidamente, ya sea las herramientas que utilizamos o en la forma en la que el público se informa" (Franco, Pellicer 2014). Además, resalta las principales habilidades que deben tener los periodistas en las redacciones de hoy.

1. Velocidad y precisión para organizar los pensamientos de forma rápida y precisa.

2. Narración visual para contar historias usando varios canales de comunicación.

3. Saber usar los medios y las redes sociales para recopilar noticias, averiguar datos, ponerse en contacto con personas y detectar tendencias, así como para distribuir el trabajo periodístico.

4. Saber valorar los datos y cómo utilizarlos para contar historias, no solo con textos, sino a través de 
gráficos y de otros recursos interactivos (Franco, Pellicer 2014).

Hoy más que nunca, el periodista debe ofrecer contenidos con valor agregado, que sobresalgan del resto de informaciones similares que se pueden encontrar en la web. Debe destacarse en ello e ir ganando el territorio que ha perdido; para esto es fundamental el desarrollo de buenas prácticas. "Evitar convertir nuestras informaciones en algo previsible, excusándose en "lo que la audiencia quiere"; evitar la observación constante de la competencia en detrimento de la realidad; evitar ser meros transmisores de la voz de los poderes fácticos; ganar el prestigio perdido con buenas prácticas y entendiendo la sociedad que nos toca vivir, así como de qué forma se ha transformado la profesión. La esencia periodística es la misma. Los instrumentos y las competencias han cambiado" (Franco, Pellicer, 2014).

Dentro del nuevo perfil se deben ir incorporando competencias multidisciplinares que abarquen el campo de lo digital y del periodismo tradicional. En lo que concierne a lo digital, como primer requisito se requiere que el periodista domine el mundo multimedia y entienda las dinámicas de la comunicación digital. Los conocimientos más técnicos también son importantes; debe entender de analítica web y algunos principios básicos de código HTML. Además, debe saber manejar su identidad digital, cómo promocionar sus contenidos en la red, filtrar la información que circula a borbotones, evaluar y verificar las fuentes digitales de valor que va a utilizar en su relato. En ese sentido, el periodista vendría a ser una especie de curador de contenidos. Es preciso mencionar también que es necesario que sepa manejar las redes sociales, tratar con un público participativo y gestionar instrumentos como microbloggins, fuentes RSS, weblogs, gestores de imágenes, archivos compartidos y visualización de datos. Para facilitar estas tareas, es necesario que el periodista cuente con una amplia cultura visual y tenga conocimientos de diseño, programación, edición y conozca sobre el tratamiento de bases de datos y de nuevos dispositivos móviles (Franco, Pellicer, 2014).

En lo que respecta a las herramientas clásicas del oficio periodístico, primero, es importante aclarar que aunque el periodismo se ha transformado, estas no han cambiado ya que son el ADN de la profesión. El olfato periodístico para detectar dónde puede estar una historia, la curiosidad y persistencia para investigar hasta encontrar lo que se busca, la sensibilidad para llegar hasta el lado humano de los hechos y conmover al público, la sagacidad y perspicacia para relacionar los rastros que van dejando los acontecimientos. A esto se suma la capacidad de análisis de los contextos y de lectura de los hechos, el manejo de fuentes, la contrastación de información; 
el tener criterio y capacidad de discernimiento para seleccionar la información. El atreverse a innovar, a plantear temas diferentes y a abordarlos desde múltiples miradas y tener una amplia cultura. Ser creativo, versátil, crítico, ético, imparcial, comprometido, actuar siempre con la verdad, ser valiente y amar lo que se hace, ante todo.

Cuando empezó el auge de los medios digitales hace más de diez años, y a la luz de los cambios producidos en torno a la producción de contenidos, se planteaba que el periodista debía ser un "hombre orquesta". En otras palabras, debía conocer un poco de todo y fusionar eficientemente sus capacidades de recolección, procesamiento y redacción de información con el manejo de herramientas digitales para elaborar contenidos multimediales y multiplataforma; ya no solo debía saber desarrollar un texto de alta calidad, con valor periodístico, sino también, tomar fotografías, grabar videos y editar audios, entre otras tareas.

Aunque, actualmente, esta realidad no ha cambiado, hoy se plantea la especialización del periodista. En estos días, el campo del conocimiento es tan amplio que es posible prepararse en un área específica para rendir con mayor eficiencia en la esfera laboral. Es así que fruto de la fusión de saberes tecnológicos y del oficio periodístico han nacido varios puestos de trabajo en las redacciones, además de los que ya existían desde algunos años como los comunity manager y analistas de tráfico. Algunos de estos son:

- Especialista en narrativas digitales: es el encargado de pensar y desarrollar nuevas maneras de contar historias, sobre todo, centradas en el campo visual.

- Periodista multimedia: es el responsable de crear piezas audiovisuales y de las posibilidades interactivas del video y la fotografía.

- Equipo de tabletas y celulares: integrado por un trío de funciones en el que se une un editor, un diseñador y un programador. Son los encargados de hacer piezas para estos dispositivos o adaptar los contenidos que ya están producidos para estos soportes.

- Periodista de datos: es una persona capaz de encontrar historias dentro de las grandes bases de datos.

En seguimiento de la clasificación que se presenta en el libro Optimismo para periodistas: claves para entender los nuevos medios de comunicación en la era digital, basado en lo que señala la periodista Lina María Aguirre Jaramillo, en la revista Capcalera, del Colegio de Periodistas de Catalunya, otros cargos presentes en varios medios internacionales son: estratega de redes sociales, optimizador de titulares, agregador de medios sociales, especialista en presentaciones digitales, curador de contenidos, reali- 
zadores y controladores virales. Estos periodistas son los encargados de crear y difundir los contenidos de una manera más efectiva en las redes sociales, gestionar los grandes archivos digitales, analizar datos, filtrar información, en definitiva, ser capaces de contar buenas historias interconectando contenidos y tecnología (Franco, Pellicer, 2014).

Otro campo que se abre para el periodista actual es el del emprendimiento. La crisis que enfrenta el sectory que fue analizada en puntos anteriores, ha llevado a que muchos periodistas emprendan su propio proyecto. El amplio campo digital ha desplegado un abanico de posibilidades en torno a la comunicación y al periodismo; proyectos creativos e innovadores a los que se les conoce como medios alternativos, los mismos que intentan recoger todo aquello que no está presente en los medios tradicionales y que se plantean como una salida a la crisis que viven las empresas informativas. Tomando en cuenta lo dicho, el periodista actual también debería fortalecer sus habilidades de emprendedor y desprenderse de la idea que las plazas de trabajo están, solamente, en los medios tradicionales.

Décadas atrás era imposible que alguien, totalmente independizado de los grandes monopolios mediáticos, pudiese tener un medio de comunicación, una voz periodística propia más allá del medio, sin embargo, hoy sí es posible.
Antes el medio era la marca, ahora lo es el periodista. Ese es, precisamente, uno de los retos para los que deben estar formados los periodistas actualmente, para trabajar alejados de las estructuras tradicionales y convertirse en una marca de calidad informativa e independencia." En la actualidad ya no existe claramente el binomio medio de comunicación-periodista. La crisis arranca a muchos periodistas de las redacciones. En otros casos, muchos profesionales entienden que trabajar para un medio de masas no es sinónimo ni de estabilidad ni de excelencia" (Franco, Pellicer, 2014).

La crisis que atraviesan las empresas informativas y que ha afectado a los periodistas es innegable; la profesión está en crisis. Para muchos esta situación está limitando el campo de acción de los periodistas e, incluso, está poniendo en duda el sentido de la profesión; sin embargo, ocurre todo lo contrario. Ahora más que nunca las posibilidades para los periodistas y comunicadores se han ampliado, la radio, la prensa, la televisión y las empresas ya no son el único lugar en los que pueden desempañarse. Desde los espacios comunitarios hasta los más avanzados proyectos transmedia necesitan periodistas y/o comunicadores. La clave para enfrentar estos nuevos escenarios está en la formación, en la construcción de un nuevo perfil multidisciplinario que integre los conocimientos y herramientas clásicas de la profesión con las nuevas tecnologías. La forma- 
ción basada en el trinomio prensa, radio y televisión hoy resulta insuficiente para abarcar todos los conocimientos y saberes que circundan el ámbito de la información y la comunicación. Es preciso pensar en un perfil más global, más integrador, que combine áreas del conocimiento que sirvan para formar profesionales que respondan a las ver- daderas necesidades de las sociedades de hoy, cambiantes, complejas, hiperinformadas pero poco formadas, y que ante todo, comprendan que aunque los años pasen y los procesos se transformen, la esencia de la vida no ha cambiado, como tampoco la esencia del periodismo. Los periodistas seguirán siendo periodistas. Punto.

\section{CONCLUSIONES}

Las empresas informativas se encuentran, permanentemente, replanteando su modelo de negocio. Las estrategias que se planifican son solo de mediano plazo, ya que no se pueden proyectar al futuro, debido a que el mercado de la información es muy cambiante. El dinamismo del entorno digital ha hecho replantear no solo la forma de producir los contenidos, sino también, las formas de monetizarlos.

Los planes de estudio de las universidades deberían estar alineados con lo que demanda el mercado laboral. Actualmente, existe una brecha entre la academia y las empresas que no permite que se puedan generar los perfiles que se requieren hoy: más amplios, versátiles y que combinen varias áreas de la profesión e, incluso, de otras. La formación ya no debería plantearse desde el trinomio prensa, radio, televisión, sino desde una perspectiva global que integre múltiples saberes relacionados con la comunicación, en la que no se pierda de vista al emprendimiento.

El nuevo perfil del periodista no solo debería contemplar conocimientos en torno a distintas áreas, sino también, integrar herramientas relacionadas al ámbito personal, life skills, que son fundamentales para el oficio periodístico. En un entono que se transforma cada día, no es suficiente requerir un profesional con unas aptitudes específicas, sino también, con unas actitudes que le permitan relacionarse adecuadamente en espacios cambiantes y complejos.

La esencia del periodismo no ha cambiado, son las herramientas las que se han transformado. Eso significa que las prácticas tradicionales del oficio siguen vigentes y continúan siendo la base de cualquier trabajo periodístico, independientemente del canal a través del que circulen los contenidos. 


\section{BIBLIOGRAFÍA}

Franco Marta, Pellicer Miquel (2014). Optimismo para periodistas: claves para entender los nuevos medios de comunicación en la era digital. Barcelona. Editorial UOC.

Noguera José Manuel, Martínez Josep, Grandío María del Mar (2011). Redes sociales para estudiantes de comunicación: 50 ideas para comprender el escenario online. Barcelona. Editorial UOC.

Canavilhas Joao (2012). Contenidos periodísticos en un ecosistema líquido: entre la convergencia y divergencia. En Irigay Fernando, Dardo Ceballos, Matías Manna (Ed.) Webperiodismo en un ecosistema líquido (pp. 11-22). Rosario: Laborde Libros Editor.

Tuñez, M., Martínez, Y., Abejón, P. (2010). Nuevos entornos, nuevas demandas, nuevos periodistas http://revistas.ucm.es/index.php /ESMP/article/view/ESMP1010110079A/11358
Punín, M.l., Martínez, A. (2013). La profesionalización periodística en Ecuador: ¿la experiencia de las calles o el conocimiento de las aulas? http://revistas.ucm.es/index.php/ ESMP/article/viewFile/42535/40444

Punín, M.I. (2012). Los estudios de comunicación social/periodismo en el Ecuador. Una visión crítica al rol de la universidad y la academia http://www.razonypalabra.org.mx /N/N79/N79/66_Punin_V79.pdf

El desempleo en el Ecuador. En sección Economía del portal Periodismo Ecuador http://periodismoecuador.com/2016/01 /19/ecuador-registra-un-desempleo-de-477en-diciembre-de-2015/

El auge de los medios nativos digitales en Ecuador. En sección Sociedad del portal PlanV http://www.planv.com.ec/historias/sociedad/el-auge-medios-nativos-digitalesecuador 